\title{
Hermitian powers: \\ A Müntz theorem and extremal algebras
}

\author{
by \\ M. J. Crabb (Glasgow), J. Duncan (Fayetteville, AR), \\ C. M. McGregor (Glasgow) and T. J. RAnsford (Québec, PQ)
}

\begin{abstract}
Given $\mathbb{S} \subset \mathbb{N}$, let $\widehat{\mathbb{S}}$ be the set of all positive integers $m$ for which $h^{m}$ is hermitian whenever $h$ is an element of a complex unital Banach algebra $A$ with $h^{n}$ hermitian for each $n \in \mathbb{S}$. We attempt to characterize when (i) $\widehat{\mathbb{S}}=\mathbb{N}$, or (ii) $\widehat{\mathbb{S}}=\mathbb{S}$. A key tool is a Müntz-type theorem which gives remarkable conclusions when $1 \in \mathbb{S}$ and $\sum\{1 / n: n \in \mathbb{S}\}$ diverges. The set $\widehat{\mathbb{S}}$ is determined by a single extremal Banach algebra $\mathrm{Ea}(\mathbb{S})$. We describe this extremal algebra for various $\mathbb{S}$.
\end{abstract}

0. Introduction. Let $A$ be a complex unital Banach algebra generated by an element $h$ and let $\mathbb{S}$ be a subset of the positive integers $\mathbb{N}$. We aim to make a general study of what can be said about $A$ given that $h^{n}$ is hermitian (that is, $\left\|\exp \left(i \alpha h^{n}\right)\right\|=1$ for all real $\alpha$ ) for $n \in \mathbb{S}$. When $\mathbb{S}=\mathbb{N}$, the Vidav-Palmer Theorem gives immediately that $A$ is a $C^{*}$-algebra with Gelfand space $\operatorname{Sp}(h)$, the spectrum of $h$. We show that the same conclusion holds under much weaker assumptions for $\mathbb{S}$. We prove in fact the following Müntz-type theorem. If $h$ is hermitian and if $h^{n}$ is hermitian for a large enough subset of odds (resp. evens) to give divergence for the corresponding series $\sum 1 / n$, then $h^{n}$ is hermitian for all odds (resp. evens). In particular, if $\mathbb{S}=3 \mathbb{N}-2$, then it follows that $h^{n}$ is hermitian for all $n \in \mathbb{N}$.

Given that $h^{n}$ is hermitian for $n \in \mathbb{S}$ in a unital Banach algebra $A$ generated by $h$, it is natural to ask if there is a largest subset $\widehat{\mathbb{S}}$ such that $h^{n}$ is hermitian for $n \in \widehat{\mathbb{S}}$ for every such unital Banach algebra $A$. This question is readily answered in the context of extremal Banach algebras. We discuss these in $\S 3$, but it is enough to note here that one definition of the extremal norm for polynomials in $h$ is given by the pointwise supremum of all algebra seminorms $|\cdot|$ such that $|h| \leq 1$ and $h^{n}$ is hermitian for all $n \in \mathbb{S}$. If a polynomial is hermitian for the extremal norm, then it is hermitian for any of these algebra seminorms. Thus we may define $\widehat{\mathbb{S}}$ to be the set of

2000 Mathematics Subject Classification: Primary 46H05. 
positive integers for which $h^{n}$ is hermitian in the extremal norm. To prove that $m \notin \widehat{\mathbb{S}}$ it is sufficient to produce any algebra seminorm for which $h^{m}$ is not hermitian.

It is natural to ask which subsets of $\mathbb{N}$ have the following properties: (i) $\widehat{\mathbb{S}}=\mathbb{N}$, (ii) $\widehat{\mathbb{S}}=\mathbb{S}$. The Müntz Theorem, which we prove in $\S 1$, enables us to identify many subsets with the first property. We conjecture that the Müntz Theorem essentially characterizes all subsets with the first property. The odds and the evens provide examples with the second property (see [CDM2]). We shed light on these two questions in $\S 2$ by constructing a series of pathological examples and thereby we determine $\widehat{\mathbb{S}}$ for many $\mathbb{S}$. In particular, we show that $\widehat{\mathbb{S}}=\mathbb{S}$ for any finite subset of $\mathbb{N}$.

In $\S 3$ we consider the extremal algebra $\mathrm{Ea}(\mathbb{S})$ for various sets $\mathbb{S}$. When $\mathbb{S}$ is a finite set $\mathbb{F}$, we have a generalization of the much studied extremal algebra $\mathrm{Ea}(\{1\})$ (see, for example, [CDM1], [CM1], [CM2]). The function theory questions associated with the general $\mathrm{Ea}(\mathbb{F})$ are rather delicate and our description is less complete than in [CDM1]. We identify the dual space as a space of entire functions in several variables with exponential growth and satisfying systems of linear complex partial differential equations. We also identify $\mathrm{Ea}(\mathbb{F})$ concretely as an algebra of operators on this space of entire functions. The brief $\S 4$ lists several open questions.

For elementary properties of hermitian elements we refer the reader to [BD].

\section{A Müntz theorem for hermitian elements}

Theorem 1.1. Let $\mathbb{S}$ be a subset of $\mathbb{N}$ such that $1 \in \mathbb{S}$.

(i) If $\sum\{1 / n: n \in \mathbb{S} \cap 2 \mathbb{N}\}=\infty$, then $\widehat{\mathbb{S}} \supset 2 \mathbb{N}$.

(ii) If $\sum\{1 / n: n \in \mathbb{S} \cap(2 \mathbb{N}-1)\}=\infty$, then $\widehat{\mathbb{S}} \supset 2 \mathbb{N}-1$.

The proof of this theorem uses two lemmas. The first is a $C^{1}$-functional calculus for hermitian elements. It is less precise but a little easier to handle than König's functional calculus $[\mathrm{K}]$. Indeed, it is possible to derive it via König's calculus, but we shall give a direct proof. In what follows, $C^{1}[a, b]$ denotes the set of functions $f:[a, b] \rightarrow \mathbb{C}$ of class $C^{1}$, which is a Banach algebra with respect to the norm

$$
\|f\|_{C^{1}[a, b]}:=\|f\|_{\infty}+\left\|f^{\prime}\right\|_{\infty} .
$$

Also, we write $u$ for the function $u(t)=t(t \in[a, b])$.

Lemma 1.1. Let $A$ be a Banach algebra and let $h$ be a hermitian element of $A$ with $\|h\| \leq 1$. Then there exists a unique continuous homomorphism $\theta: C^{1}[-1,1] \rightarrow A$ such that $\theta(u)=h$. 
Proof. Uniqueness is clear, since polynomials are dense in $C^{1}[-1,1]$. For existence, it is enough to show that there exists a constant $C$ such that, for every polynomial $p$,

$$
\|p(h)\| \leq C\|p\|_{C^{1}[-1,1]} .
$$

Indeed, if (1) holds, then $\theta$, defined on polynomials by $\theta(p)=p(h)$, is continuous with respect to $\|\cdot\|_{C^{1}[-1,1]}$, and so it extends by continuity to the whole of $C^{1}[-1,1]$.

We shall prove that $(1)$ holds with $C=\sqrt{7 / 3}$. Let $\varphi$ be a continuous linear functional on $A$ such that $\|\varphi\|=1$. Define $F(z)=\varphi\left(e^{i z h}\right)(z \in \mathbb{C})$. Then $F$ is an entire function of exponential type, in fact,

$$
|F(z)| \leq\|\varphi\| \cdot\left\|e^{i(\operatorname{Re} z) h}\right\| \cdot\left\|e^{-(\operatorname{Im} z) h}\right\| \leq e^{|\operatorname{Im} z|} \quad(z \in \mathbb{C}) .
$$

Define $G(z)=(F(z)-F(0)) / z(z \in \mathbb{C})$. Then $G$ is also an entire function of exponential type and $|G(x)| \leq 2 /|x|$ for $x \in \mathbb{R}, x \neq 0$. Thus $G \in L^{2}(\mathbb{R})$. By the Paley-Wiener Theorem, there exists $g \in L^{2}[-1,1]$ such that

$$
G(z)=\int_{-1}^{1} g(t) e^{i t z} d t \quad(z \in \mathbb{C}),
$$

and by Parseval's theorem

$$
\int_{-1}^{1}|g(t)|^{2} d t=\frac{1}{2} \sum_{n \in \mathbb{Z}}|G(n \pi)|^{2}
$$

Since $|G(0)|=\left|F^{\prime}(0)\right| \leq 1$ and $|G(n \pi)| \leq 2 / n \pi$ for $n \neq 0$, it follows that

$$
\int_{-1}^{1}|g(t)|^{2} d t \leq \frac{1}{2}+\frac{4}{\pi^{2}} \sum_{n=1}^{\infty} \frac{1}{n^{2}}=\frac{1}{2}+\frac{2}{3}=\frac{7}{6} .
$$

Rewriting in terms of $F$, we have

$$
F(z)=F(0)+z \int_{-1}^{1} g(t) e^{i t z} d t \quad(z \in \mathbb{C}) .
$$

It follows that, for $n \geq 1$,

$$
F^{(n)}(0)=\int_{-1}^{1} g(t) n(i t)^{n-1} d t
$$

Now let $p$ be a polynomial, say $p(t)=\sum_{n=0}^{N} a_{n} t^{n}$. Then

$$
\varphi(p(h)-p(0))=\sum_{n=1}^{N} a_{n} \varphi\left(h^{n}\right)=\sum_{n=1}^{N} a_{n} i^{-n} F^{(n)}(0)=\int_{-1}^{1} i^{-1} g(t) p^{\prime}(t) d t .
$$


Therefore, by Schwarz's inequality,

$$
|\varphi(p(h)-p(0))| \leq\left(\int_{-1}^{1}|g(t)|^{2} d t\right)^{1 / 2}\left(\int_{-1}^{1}\left|p^{\prime}(t)\right|^{2} d t\right)^{1 / 2} \leq \sqrt{7 / 6} \sqrt{2}\left\|p^{\prime}\right\|_{\infty} .
$$

Since $\varphi$ is an arbitrary linear functional on $A$ of norm 1, this implies that

$$
\|p(h)-p(0)\| \leq \sqrt{7 / 3}\left\|p^{\prime}\right\|_{\infty},
$$

and this in turn implies that (1) holds with $C=\sqrt{7 / 3}$.

The second lemma is a mild generalization of the classical Müntz Theorem.

Lemma 1.2 (Müntz for $C^{1}[0,1]$ ). Let $\mathbb{S} \subset \mathbb{N}$ be such that $1 \in \mathbb{S}$ and $\sum_{n \in \mathbb{S}} 1 / n=\infty$. Then the span of $\left\{1, t^{n}(n \in \mathbb{S})\right\}$ is dense in $C^{1}[0,1]$.

Proof. Let $\varphi$ be a continuous linear functional on $C^{1}[0,1]$ that annihilates $\left\{1, t^{n}(n \in \mathbb{S})\right\}$. Consider $F(z)=\varphi\left(t^{z}\right) / z(\operatorname{Re} z>1)$. This is bounded and analytic and vanishes on $\mathbb{S}$. Transfer to a bounded analytic function on the open unit disk by taking $G(z)=F(2 /(1-z))$. Then $G$ has zeros at $1-2 / n$ for $n \in \mathbb{S}$. It follows from [R], Theorem 15.23 , that $G \equiv 0$, and hence also $F \equiv 0$. This gives $\varphi\left(t^{z}\right)=0(\operatorname{Re} z>1)$ and so $\varphi=0$. The Hahn-Banach Theorem completes the proof.

Proof of Theorem 1.1. We prove the odd case; the even case is similar. Let $k$ be an odd positive integer. By Lemma 1.2 there exist polynomials $p_{n}(t)$ with powers of $t$ only from $\mathbb{S} \cap(2 \mathbb{N}-1)$ such that $p_{n}(t)$ converges to $t^{k}$ in $C^{1}[0,1]$. Since all these functions are odd, we actually have convergence in $C^{1}[-1,1]$. Moreover, replacing $p_{n}$ by $\operatorname{Re} p_{n}$, we can suppose that the $p_{n}$ have real coefficients. Let $A$ be a Banach algebra that contains the hermitian element $h$. Without loss of generality we may suppose that $|h|=1$. By Lemma 1.1, $p_{n}(h)$ converges to $h^{k}$ in $A$. Since $p_{n}(h)$ is hermitian, it follows that $h^{k}$ is hermitian.

2. Some determinations of $\widehat{\mathbb{S}}$. The above Müntz Theorem has many immediate consequences. We state some as a theorem.

TheOREM 2.1. Let $h$ be a hermitian element of a complex unital Banach algebra $A$.

(i) If $h^{n}$ is hermitian for $n$ in an arithmetic progression which is contained within neither the evens nor the odds, then $h^{n}$ is hermitian for all $n \in \mathbb{N}$.

(ii) If $h^{n}$ is hermitian for $n$ in a cofinite subset of $\mathbb{N}$, then $h^{n}$ is hermitian for all $n \in \mathbb{N}$.

(iii) If $h^{n}$ is hermitian for every odd prime, then $h^{n}$ is hermitian for every odd $n$. 
When $\mathbb{S}$ satisfies either (i) or (ii) in the above theorem, it is immediate that $\widehat{\mathbb{S}}=\mathbb{N}$. Now let $\mathbb{S}=2 \mathbb{N}-1$. It follows from [CDM2] that $\widehat{\mathbb{S}}=2 \mathbb{N}-1$. The same conclusion holds for $\mathbb{S}=4 \mathbb{N}-3,6 \mathbb{N}-5, \ldots$ Now let $\mathbb{S}=2 \mathbb{N}$. It follows from [CDM2] that $\widehat{\mathbb{S}}=\mathbb{S}$ and we shall see in the next section that the same conclusion holds for $\mathbb{S}=m \mathbb{N}$ for any $m \geq 2$.

From the viewpoint of the Müntz Theorem, there is not an exact parallel between the odds and the evens. The natural analogue for the evens is given by $\mathbb{S}=\{1\} \cup 2 \mathbb{N}$, and it is by no means immediate that $\widehat{\mathbb{S}}=\mathbb{S}$ for this case. We prove this by constructing an example in which $h^{n}$ is hermitian precisely for such $n$. Let $\mathcal{F}$ be the real linear span of the functions $t^{n}$ for $n \in \mathbb{S}$, and let $G=\exp (i \mathcal{F})$. Let $X=C[-1,1]$ with the norm defined by

$$
\|x\|=\sup _{g \in G}\left|\int_{-1}^{1} g(t) x(t) d t\right|
$$

and let $T$ be the linear operator given by $T x(t)=t x(t)$. The function $t$, appropriately extended, has an absolutely convergent Fourier series representation on $[-2,2]$. It follows that $T$ is a bounded operator.

Proposition 2.1. For the above operator $T$, we have $T^{n}$ hermitian for $n \in\{1\} \cup 2 \mathbb{N}$ and not hermitian for $n$ odd, $n \geq 3$.

Proof. We easily check that, for $n \in\{1\} \cup 2 \mathbb{N}$,

$$
\left\|\exp \left(i \alpha T^{n}\right) x\right\|=\|x\| \quad(\alpha \in \mathbb{R})
$$

and so $T^{n}$ is hermitian. Suppose that $T^{2 k+1}$ is hermitian for some $k \in \mathbb{N}$. Let $v(t)=1$. Since $\left\|\exp \left(i T^{2 k+1}\right) v\right\|=\|v\|$, we have

$$
\sup _{g \in G}\left|\int_{-1}^{1} \exp \left(i t^{2 k+1}\right) g(t) d t\right|=2 .
$$

By compactness, we get a sequence of functions $g$ so that the integral converges to some $2 \exp (i \beta)$. By absorbing $\beta$ into $g$, we thus get sequences of reals $a_{n}$ and even real functions $\xi_{n}(t)$ such that

$$
\lim \int_{-1}^{1} \exp \left(i\left(t^{2 k+1}+a_{n} t+\xi_{n}(t)\right)\right) d t=2 .
$$

Conjugate this equation and replace $t$ by $-t$ to conclude that

$$
\lim \int_{-1}^{1} \cos \left(t^{2 k+1}+a_{n} t\right) \cos \left(\xi_{n}(t)\right) d t=2 .
$$


It follows from the Cauchy-Schwarz inequality that $\lim J\left(a_{n}\right)=2$ where

$$
J(a)=\int_{-1}^{1} \cos ^{2}\left(t^{2 k+1}+a t\right) d t .
$$

Clearly, $|J(a)|<2$ for any real $a$. The Riemann-Lebesgue Lemma shows that $J(a) \rightarrow 1$ as $|a| \rightarrow \infty$. This contradiction completes the proof.

It is immediate that $\widehat{\mathbb{S}}=\mathbb{S}$ for $\mathbb{S}=\{1\} \cup 2 \mathbb{N}$. The above example is, in a sense, a universal example since we may replace $d t$ by an arbitrary positive measure $d \mu$. By taking $d \mu$ to be a finite linear combination of point masses we can produce finite-dimensional examples. The following example may be the simplest possible with $\mathbb{S}=\{1\} \cup 2 \mathbb{N}$.

Proposition 2.2. Let $T$ be the diagonal operator on $\mathbb{C}^{5}$ with entries $(-2,-1,0,1,2)$ and let $\mathbb{C}^{5}$ have the norm

$$
\|x\|=\max _{z, w, t \in \mathbb{T}}\left|z t^{-2} x_{-2}+w t^{-1} x_{-1}+x_{0}+w t x_{1}+z t^{2} x_{2}\right| .
$$

Then $T$ has the same property as the operator in Proposition 2.1.

Proof. Note that $\exp \left(i \alpha T^{n}\right)$ is also diagonal with entries $\exp \left(i \alpha j^{n}\right)$ where $j=-2,-1,0,1,2$. It follows easily that $T^{n}$ is hermitian for $n=1$ and for any even positive integer. Suppose that $T^{3}$ is hermitian and let $v=(1,1,1,1,1)$. Then $\left\|\exp \left(i \alpha T^{3}\right) v\right\|=\|v\|=5$ for all real $\alpha$. It follows that, for each real $\alpha$, there exist $z, w, t \in \mathbb{T}$ such that

$$
z t^{-2} e^{-8 i \alpha}=w t^{-1} e^{-i \alpha}=w t e^{i \alpha}=z t^{2} e^{8 i \alpha}=1 .
$$

Eliminate $z, w$ to give $e^{16 i \alpha}=t^{-4}$ and $e^{2 i \alpha}=t^{-2}$. It follows that $e^{16 i \alpha}=e^{4 i \alpha}$ for all real $\alpha$. This contradiction shows that $T^{3}$ is not hermitian, and a similar argument gives $T^{n}$ not hermitian for any odd $n \geq 3$.

To get a finite-dimensional example for which $T^{n}$ is hermitian if and only if $n=1,3,2 m(m \in \mathbb{N})$, we take the diagonal operator with entries

$$
(-3,-2,-1,0,1,2,3)
$$

and norm

$$
\|x\|=\max _{u, v, w, s, t \in \mathbb{T}}\left|u s^{-27} t^{-3} x_{-3}+v s^{-8} t^{-2} x_{-2}+w s^{-1} t^{-1} x_{-1}+x_{0}+\ldots\right| .
$$

An elaboration handles the case $\mathbb{S}=\mathbb{F} \cup 2 \mathbb{N}$ where $\mathbb{F}$ is a finite subset of the odds which contains 1 . It is instructive to consider a slightly simpler construction for which we obtain a slightly stronger conclusion for $\mathbb{S}=\mathbb{F}$. 
Proposition 2.3. Let $\mathbb{S}=\left\{m_{j}: j=1, \ldots, q\right\}$ with $m_{1}<\ldots<m_{q}$. Let $N \geq m_{q}+1$, and let $X=\mathbb{C}^{2 N+1}$ with norm defined by

$$
\|x\|=\max _{z_{k} \in \mathbb{T}}\left|x_{-N} \prod_{k=1}^{q} z_{k}^{(-N)^{m_{k}}}+\ldots+x_{0}+\ldots+x_{N} \prod_{k=1}^{q} z_{k}^{N^{m_{k}}}\right| .
$$

Let $T$ be the diagonal operator on $X$ with entries

$$
(-N, \ldots,-1,0,1, \ldots, N) .
$$

Then $T^{m}$ is hermitian for $m \in \mathbb{S}$ and the space of hermitians in the subalgebra generated by $T$ is just the real linear span of $I$ and $T^{m}$ for $m \in \mathbb{S}$.

Proof. Note that $\exp \left(i \alpha T^{m_{k}}\right)$ is also diagonal, with entries

$$
\left(\exp \left(i \alpha(-N)^{m_{k}}\right), \ldots, 1, \ldots, \exp \left(i \alpha(N)^{m_{k}}\right)\right) .
$$

It follows from the definition of the norm that

$$
\left\|\exp \left(i \alpha T^{m_{k}}\right) x\right\|=\|x\|
$$

for all real $\alpha$, and so $T^{m_{k}}$ is hermitian for $k=1, \ldots, q$. The algebra generated by $T$ consists of polynomials of degree at most $2 N$. Let $P(T)=\sum_{j=1}^{\nu} c_{j} T^{n_{j}}$ be such a polynomial with $n_{j} \neq m_{k}$ for any $j, k$, and suppose that $P(T)$ is hermitian. Then $P(T)$ has real spectrum and so $P(j)$ is real for $j=$ $-N, \ldots, N$. Hence the coefficients $c_{j}$ are all real. Let

$$
U=\exp (i \alpha P(T))=\prod_{j} \exp \left(i \alpha c_{j} T^{n_{j}}\right)
$$

and let $v=(1, \ldots, 1)$ so that $\|v\|=2 N+1$. We show that $\|U v\|=2 N+1$ leads to a contradiction unless $P(T)=0$. Since $U$ is diagonal with entries from $\mathbb{T}$, there must exist $z_{j} \in \mathbb{T}$ so that each term in the norm expression for $U v$ has value 1 . Thus

$$
z_{1}^{j^{m_{1}}} \ldots z_{q}^{j^{m_{q}}} \exp \left(i \alpha c_{1} j^{n_{1}}\right) \ldots \exp \left(i \alpha c_{\nu} j^{n_{\nu}}\right)=1
$$

for $j= \pm 1, \ldots, \pm N$. Write $z_{j}=\exp \left(i \theta_{j}\right)$ to get

$$
\theta_{1} j^{m_{1}}+\ldots+\theta_{q} j^{m_{q}}+\alpha c_{1} j^{n_{1}}+\ldots+\alpha c_{\nu} j^{n_{\nu}} \in 2 \pi \mathbb{Z}
$$

for $j= \pm 1, \ldots, \pm N$. But this contains an invertible Vandermonde system of linear equations and the inverse matrix has rational entries. Hence $\alpha c_{j} \in 2 \pi \mathbb{Q}$ for all real $\alpha$. This forces all $c_{j}=0$ and then $P(T)$ is the zero polynomial, as required.

TheOREM 2.2. We have $\widehat{\mathbb{F}}=\mathbb{F}$ for any finite subset of $\mathbb{N}$.

We also observe that pathological behaviour can occur even for finitedimensional Hilbert space operators when the generator is not hermitian. 
EXAMPLE 2.1. Let $T$ be the operator on three-dimensional Hilbert space given by

$$
T=\left[\begin{array}{lll}
1 & 0 & 0 \\
0 & 0 & c \\
0 & 0 & 0
\end{array}\right]
$$

Then, for any $c \in \mathbb{C}, T^{n}$ is hermitian for $n \geq 2$ but the algebra generated by $T$ is not semisimple. Note also that there is no uniform bound on $|T|$ even though all these hermitian powers have numerical range equal to $[0,1]$.

3. Some determinations of $\mathrm{Ea}(\mathbb{S})$. There are several ways to define extremality, but the most convenient is in terms of the polynomial functional calculus. We restrict attention here to algebras with a single generator $h$ and to the property $\tau$ that $h^{n}$ be hermitian for every $n \in \mathbb{S}$. Let $\mathbb{C}[h]$ be the algebra of all complex polynomials in $h$. We say that a unital singly generated Banach algebra $(A,\|\cdot\|)$ is extremal for $\tau$ if $A$ has property $\tau$ and if $|P(h)| \leq\|P(h)\|(P \in \mathbb{C}[h])$ for every unital singly generated Banach algebra $(B,|\cdot|)$ with property $\tau$. The existence of an extremal Banach algebra immediately forces uniqueness. We cannot have existence without a uniform bound on $|h|$. In [CDM1] this uniform bound was guaranteed by imposing the condition that the generator have numerical range within the closed unit disk. In the present context, it might seem natural to require that the numerical range of each hermitian $h^{n}$ lie within the unit interval $[-1,1]$. When $1 \notin \mathbb{S}$, this does not guarantee a uniform bound on $|h|$ (see Example 2.1). Accordingly, we shall always require that the generator have norm at most 1 .

Suppose now that $\sigma$ is the property that $|h| \leq 1$ and $h^{n}$ is hermitian for $n \in \mathbb{S}$ for a singly generated Banach algebra $(B,|\cdot|)$. We can guarantee the existence of the corresponding extremal algebra $\mathrm{Ea}(\mathbb{S})$ by construction. For $|k| \leq 1$, we have $k$ hermitian if and only if

$$
|\cos \theta+i \sin \theta k| \leq 1 \quad(\theta \in \mathbb{R}) .
$$

(This follows easily from the characterization, $|1+i t k|=1+o(t)$ and Sinclair's theorem $|\cos \theta+i \sin \theta \quad k|=r(\cos \theta+i \sin \theta k)$ for hermitian $k$.) Let $S$ be the semigroup in $\mathbb{C}[h]$ generated by all expressions of the form $\cos \theta+i \sin \theta h^{n}$ for $\theta \in \mathbb{R}$ and $n \in \mathbb{S}$. Define a unital algebra seminorm on $\mathbb{C}[h]$ by

$$
\|P(h)\|=\inf \left\{\sum_{j=1}^{N}\left|\alpha_{j}\right|: N \in \mathbb{N}, \alpha_{j} \in \mathbb{C}, s_{j} \in S, \sum_{j=1}^{N} \alpha_{j} s_{j}=P(h)\right\} .
$$

It is immediate that $|P(h)| \leq\|P(h)\|$ for any singly generated Banach algebra $(B,|\cdot|)$ with property $\sigma$. This holds in particular when

$$
|P(h)|=\sup \{|P(t)|: t \in[-1,1]\}
$$


and so $\|\cdot\|$ is an algebra norm. Then $\mathrm{Ea}(\mathbb{S})$ is the completion of $\mathbb{C}[h]$ with respect to $\|\cdot\|$. Also, $\widehat{\mathbb{S}}=\left\{n \in \mathbb{N}: h^{n}\right.$ is hermitian in $\left.\mathrm{Ea}(\mathbb{S})\right\}$ gives the largest set of hermitian powers of $h$ given that $h^{n}$ is hermitian for all $n \in \mathbb{S}$. The constraint that $h^{n}$ be hermitian for all $n \in \mathbb{S}$ gives a condition on the norm only for elements in the subalgebra generated by 1 and $h^{n}$ for $n \in \mathbb{S}$. In general, this subalgebra is not all of $\mathbb{C}[h]$. The only constraint on all of $\mathbb{C}[h]$ is that $\|h\| \leq 1$.

There is an alternative construction of the norm $\|\cdot\|$ by taking the pointwise supremum of all unital algebra seminorms on $\mathbb{C}[h]$ such that $|h| \leq 1$ and $h^{n}$ is hermitian for all $n \in \mathbb{S}$. We need to work with seminorms to include the case when $h$ satisfies a polynomial identity; fortunately, the elementary ideas of numerical ranges work just as well for seminorms. Every such seminorm is dominated by the $\ell^{1}$ norm on $\mathbb{C}[h]$ and so the supremum exists. For $\|\cdot\|$ to be a norm, it is sufficient to have one norm with property $\sigma$.

We need discuss $\mathrm{Ea}(\mathbb{S})$ only for subsets of $\mathbb{N}$ which are an $\widehat{\mathbb{S}}$. We shall do so for the cases $\mathbb{S}=\mathbb{N}, 2 \mathbb{N}-1, m \mathbb{N},\{1\} \cup 2 \mathbb{N}$ and $\mathbb{F}$, where $\mathbb{F}$ is a finite set. We have no information for subsets of the odds, or the evens, for which the corresponding harmonic series converges, for example, $\mathbb{S}=\left\{(2 n-1)^{2}\right.$ : $n \in \mathbb{N}\}$.

Evidently, $\mathrm{Ea}(\mathbb{N})$ is isometrically isomorphic to $C[-1,1]$. It is shown in [CDM3] that $\mathrm{Ea}(2 \mathbb{N}-1)$ is bicontinuously isomorphic to $C[-1,1]$. There is thus a surprisingly large collection of subsets $\mathbb{S}$ of $\mathbb{N}$ for which $\mathrm{Ea}(\mathbb{S})$ is $C^{*}$-equivalent.

The extremal algebra $\operatorname{Ea}(2 \mathbb{N})$ is not $C^{*}$-equivalent. To see this, recall from [CDM2] that the Gelfand space of $\mathrm{Ea}(2 \mathbb{N})$ is the cross $K=[-1,1] \cup$ $[-i, i]$, and $\mathrm{Ea}(2 \mathbb{N})$ separates the points of $K$. If $\mathrm{Ea}(2 \mathbb{N})$ were $C^{*}$-equivalent, then it would be all of $C(K)$. One may complete the proof by showing that not every $f \in C(K)$ may be written in the form $f(t)=f_{0}(t)+t f_{1}(t)$ with $f_{0}, f_{1}$ even functions on $K$. It is instructive to give another proof. The mapping $f \mapsto f_{1}(0)$ is readily verified to be a non-zero bounded point derivation (at $t=0$ ) and hence $\mathrm{Ea}(2 \mathbb{N}$ ) is not an amenable Banach algebra and therefore not $C^{*}$-equivalent.

We now generalize to the case $\mathbb{S}=m \mathbb{N}$ where $m$ is an arbitrary positive integer. Let $\omega$ be a primitive $m$ th root of unity and let $K_{m}$ be the regular star defined by

$$
K_{m}=\bigcup_{j=0}^{m-1} \omega^{j}[-1,1] .
$$

Now let $A$ be any complex unital Banach algebra generated by $h$ with $\|h\|$ $=1$ and $h^{n}$ hermitian for $n \in m \mathbb{N}$. Let $k=h^{m}$. Then $k^{n}$ is hermitian for $n \in \mathbb{N}$, and so the Vidav-Palmer Theorem gives $\langle k\rangle=C(\operatorname{Sp}(k))$, where $\langle k\rangle$ 
is the closed subalgebra generated by $k$. In particular, any polynomial in $h^{m}$ has norm equal to the spectral radius (denoted by $r(\cdot)$ ). Any polynomial $P(h)$ may be written as

$$
P(h)=\sum_{j=0}^{m-1} h^{j} P_{j}\left(h^{m}\right)
$$

for (unique) polynomials $P_{j}$. Since $\|h\|=1$, we get

$$
\|P(h)\| \leq \sum_{j=0}^{m-1}\left\|P_{j}\left(h^{m}\right)\right\|=\sum_{j=0}^{m-1} r\left(P_{j}\left(h^{m}\right)\right) .
$$

A routine calculation shows that the latter formula gives an algebra norm on the polynomial algebra such that $h^{n}$ is hermitian for $n \in m \mathbb{N}$. The extremal algebra $\mathrm{Ea}(m \mathbb{N})$ is just the completion when $\operatorname{Sp}(h)=K_{m}$.

As in [CDM2], we may readily identify $\operatorname{Ea}(m \mathbb{N})$ with those continuous functions $f$ on the regular star, $K_{m}$, such that, for $j=1, \ldots, m-1$,

$$
\lim _{t \rightarrow 0} \frac{1}{t^{m-j}}\left[f(t)+\omega^{j} f(\omega t)+\ldots+\omega^{(m-1) j} f\left(\omega^{m-1} t\right)\right]
$$

exists. Equivalently, we get the algebra $\mathcal{E}_{m}$ of all continuous complex functions on $K_{m}$ of the form

$$
f(t)=f_{0}(t)+t f_{1}(t)+\ldots+t^{m-1} f_{m-1}(t)
$$

where each $f_{j}$ is a continuous function on $K_{m}$ invariant under the cyclic action on $K_{m}$. The norm on $\mathcal{E}_{m}$ is given by

$$
\|f\|=\sum_{j=0}^{m-1} r\left(f_{j}\right) .
$$

As above, we get a non-zero bounded point derivation on $\mathcal{E}_{m}$ defined by $f \mapsto f_{1}(0)$. In particular, $\mathcal{E}_{m}$ is not an amenable Banach algebra and is not $C^{*}$-equivalent (for $m>1$ ). It is also straightforward to check that $V\left(h^{n}\right)=\mathbb{D}^{-}$(the closed unit disk) when $n \notin m \mathbb{N}$. We summarize these facts in a theorem.

TheOREM 3.1. For $m>1, \mathrm{Ea}(m \mathbb{N})$ is isometrically isomorphic to $\mathcal{E}_{m}$ and is not $C^{*}$-equivalent. We also have $(\widehat{m \mathbb{N}})=m \mathbb{N}$.

Note that the generator $h$ for $\mathrm{Ea}(2 \mathbb{N})$ is not hermitian. We make it hermitian by taking $\mathbb{S}=\{1\} \cup 2 \mathbb{N}$. Since $h$ is hermitian, we have $\operatorname{Sp}(h) \subset$ $[-1,1]$. It is tempting to think that $\mathrm{Ea}(\mathbb{S})$ might be the algebra of functions $\mathcal{E}_{2}$ restricted from the cross $K$ to the interval $[-1,1]$. This cannot be so, since the element $h$ is not hermitian for the $\mathcal{E}_{2}$ norm. But the argument in 
Theorem 4.2 of [CDM2] shows that the norm in $\mathrm{Ea}(\mathbb{S})$ is dominated by this restricted $\mathcal{E}_{2}$ norm.

We turn finally to the case of a finite subset $\mathbb{F}$ of $\mathbb{N}$, beginning with the case in which $\mathbb{F}$ is a singleton, say, $\mathbb{F}=\{m\}$. For $m=1$, we have, of course, $\mathrm{Ea}(\mathbb{F})=\mathrm{Ea}[-1,1]$ (in the notation of [CDM1]). For arbitrary $m$, let $k=h^{m}$ and clearly $\langle k\rangle=\mathrm{Ea}[-1,1]$. Decompose an arbitrary polynomial $P(h)$ as above to define

$$
\|P(h)\|=\sum_{j=0}^{m-1}\left\|P_{j}\left(h^{m}\right)\right\|_{0}
$$

where $\|\cdot\|_{0}$ is the norm from $\mathrm{Ea}[-1,1]$ transferred by $k=h^{m}$. Again, it is easy to check that this gives a norm and the completion is the required extremal algebra. As before, we have $\operatorname{Sp}(h)=K_{m}$, and we may represent $\mathrm{Ea}(\mathbb{F})$ as an algebra of continuous functions on $K_{m}$. The $\ell^{1}$ nature of the norm gives that $V\left(h^{n}\right)=\mathbb{D}^{-}$when $n \notin m \mathbb{N}$ and it follows that $h^{m}$ is the only positive power of $h$ which is hermitian in the extremal algebra, that is, $\widehat{\mathbb{F}}=\mathbb{F}$ (as already proved by a different method in Theorem 2.2).

While it is possible to give an (inelegant) description of $\mathrm{Ea}(\mathbb{F})$ for general $\mathbb{F}$ with $1 \notin \mathbb{F}$, for simplicity we shall restrict attention to the case in which $1 \in \mathbb{F}$.

Suppose then that $1 \in \mathbb{F}$. We shall give an explicit description of the dual space, $\Phi_{\mathbb{F}}$, of $\mathrm{Ea}(\mathbb{F})$ and this will lead to an explicit representation of $\mathrm{Ea}(\mathbb{F})$ as an algebra of operators on $\Phi_{\mathbb{F}}$. Since the argument is a little complicated, we shall begin with the simplest possible example, namely $\mathbb{F}=\{1,2\}$, and then we shall discuss how to modify the argument for the general case.

Recall from above that $S$ is the multiplicative subsemigroup of $\mathbb{C}[h]$ consisting of all finite products of elements of the form $\cos \theta+i \sin \theta h^{j}$ where $j \in \mathbb{S}$ and $\theta \in \mathbb{R}$. For $P(h) \in \mathbb{C}[h]$ we have

$$
\|P(h)\|=\inf \left\{\sum\left|\alpha_{n}\right|: P(h)=\sum \alpha_{n} \pi_{n}, \alpha_{n} \in \mathbb{C}, \pi_{n} \in S\right\} .
$$

This gives the norm on $\mathbb{C}[h]$ whose completion is $\mathrm{Ea}(\mathbb{S})$.

For the case $\mathbb{F}=\{1,2\}$, we write $\Phi$ for the set of all entire functions $\varphi$ in two complex variables such that, for some $M_{\varphi}$,

$$
|\varphi(z, w)| \leq M_{\varphi} \varpi(z) \varpi(w), \quad \frac{\partial \varphi}{\partial w}=\frac{\partial^{2} \varphi}{\partial z^{2}}
$$

where $\varpi(z)=\exp |\operatorname{Re} z|$, and let

$$
\|\varphi\|=\sup \left\{\frac{|\varphi(z, w)|}{\varpi(z) \varpi(w)}: z, w \in \mathbb{C}\right\} .
$$

It is routine to show that $\Phi$ is a Banach space. 
TheOREM 3.2. The dual space of $\mathrm{Ea}(\mathbb{F})$ is isometrically isomorphic to $\Phi$.

Proof. Given $\varphi \in \Phi$, let $F_{\varphi}$ be the linear functional defined on $\mathbb{C}[h]$ by

$$
F_{\varphi}\left(h^{n}\right)=\frac{\partial^{a+b} \varphi}{\partial z^{a} \partial w^{b}}(0,0)
$$

where $n=a+2 b$. This is well defined because of the partial differential equation satisfied by $\varphi$. To prove that $F_{\varphi}$ is continuous with $\left\|F_{\varphi}\right\| \leq\|\varphi\|$ it is enough to prove that $\left|F_{\varphi}(\pi(h))\right| \leq\|\varphi\|$ for each $\pi \in S$. We have

$$
F_{\varphi}(\cos \theta+i \sin \theta h)=\cos \theta \varphi(0,0)+i \sin \theta \frac{\partial \varphi}{\partial z}(0,0)
$$

and

$$
F_{\varphi}\left(\cos \theta+i \sin \theta h^{2}\right)=\cos \theta \varphi(0,0)+i \sin \theta \frac{\partial \varphi}{\partial w}(0,0)
$$

and the required inequalities are immediate by the classical Bernstein theorem for entire functions of exponential type. Suppose now that the inequality holds when $\pi(h)$ is the product of $n$ factors. Now, for all $n$, define $G\left(h^{n}\right)=F_{\varphi}\left((\cos \theta+i \sin \theta h) h^{n}\right)$. An easy computation shows that $G=F_{\psi}$ where $\psi=\cos \theta \varphi+i \sin \theta \partial \varphi / \partial z$. We now have

$$
F_{\varphi}((\cos \theta+i \sin \theta h) \pi(h))=F_{\psi}(\pi(h)) .
$$

The induction hypothesis and Bernstein's theorem now extend the required inequality to the above $n+1$ factors. A similar argument applies when the new factor is of the form $\cos \theta+i \sin \theta h^{2}$ when we use the partial derivative with respect to $w$. We now see that $\varphi \mapsto F_{\varphi}$ is a monomorphism from $\Phi$ into $\mathrm{Ea}(\mathbb{S})^{\prime}$. Suppose now that $F \in \mathrm{Ea}(\mathbb{S})^{\prime}$. Since $h, h^{2}$ are hermitian and $\|h\| \leq 1$, we know that $\left\|\exp \left(z h+w h^{2}\right)\right\| \leq \varpi(z) \varpi(w)$. We define $\varphi$ by

$$
\varphi(z, w)=F\left(\exp \left(z h+w h^{2}\right)\right)
$$

and we readily verify that $\varphi \in \Phi$ and $F=F_{\varphi}$. The above formula also gives $\|F\| \geq\|\varphi\|$ and so the isometric isomorphism is established. It is easy to check that the Gelfand space of $\mathrm{Ea}(\mathbb{S})$ corresponds to the entire functions $\exp \left(t z+t^{2} w\right)$ indexed by $t \in[-1,1]$.

We constructed $\mathrm{Ea}(\mathbb{S})$ as an abstract completion, but the method of Browder (see [B1], [B2]) allows us to give a specific representation for $\mathrm{Ea}(\mathbb{S})$. Let $T$ be the differential operator on $\Phi$ defined by $T=\partial / \partial z$. Then $\mathrm{Ea}(\mathbb{S})$ is isometrically isomorphic to the closed subalgebra of $B(\Phi)$ generated by the hermitian operator $T$.

REMARKs. (1) Since $V\left(h^{2}\right) \subset[0,1]$, we have the sharper inequality

$$
\left\|\exp \left(w h^{2}\right)\right\| \leq \varpi_{+}(w)
$$


where $\varpi_{+}(w)=\max (\exp (\operatorname{Re} w), 1)$ and so each $\varphi \in \Phi$ satisfies the sharper inequality

$$
|\varphi(z, w)| \leq \varpi(z) \varpi_{+}(w) .
$$

A standard Phragmén-Lindelöf argument also shows that the norm of $\varphi$ in $\Phi$ is determined by taking the supremum over $i \mathbb{R} \times i \mathbb{R}$.

(2) The exact computation of $V(P(h))$ now becomes a delicate coefficient problem. We may illustrate this adequately by considering the case $P(h)$ $=h^{3}$. A routine power series argument shows that any $\varphi \in \Phi$ is uniquely determined by the entire function $g(z)=\varphi(z, 0)$. In fact, we have

$$
\varphi(z, w)=g(z)+g^{\prime \prime}(z) w+g^{(4)}(z) w^{2} / 2 !+\ldots
$$

Since

$$
F_{\varphi}\left(h^{3}\right)=\frac{\partial^{3} \varphi}{\partial z^{3}}(0,0)=g^{\prime \prime \prime}(0)
$$

it follows that

$$
V\left(h^{3}\right)=\left\{g^{\prime \prime \prime}(0): g(0)=1=\|\varphi\|\right\} .
$$

Given any entire $g(z)$ with $g(0)=1$ and $|g(z)| \leq e^{|\operatorname{Re} z|}$, Bernstein's Theorem gives the same growth condition for all derivatives of $g$. The power series formula for $\varphi(z, w)$ immediately satisfies $|\varphi(z, w)| \leq e^{|\operatorname{Re} z|+|w|}$. By Phragmén-Lindelöf, this $\varphi$ gives a support functional at the identity of $\mathrm{Ea}(\mathbb{S})$ if and only if $|\varphi(i y, i v)| \leq 1$ for all real $y, v$. Herein lies the delicacy in calculating $V\left(h^{n}\right)$. It is not even immediately obvious that $h^{n}$ fails to be hermitian for $n \geq 3$ in this case. This can be shown by the method of [CDM1] and the theory of oscillatory integrals, but it was simpler to do so by the method of Section 2 .

(3) By analogy with [CDM1] we should expect to be able to identify $\mathrm{Ea}(\mathbb{F})$ (for the present example) with all functions on $[-1,1]$ of the form

$$
f(t)=\int_{\mathbb{C}^{2}} \exp \left(t z+t^{2} w\right) d \mu(z, w)
$$

where $\mu$ is any regular Borel measure on $\mathbb{C}^{2}$ with

$$
\int_{\mathbb{C}^{2}} \varpi(z) \varpi(w) d|\mu|<\infty .
$$

The key step in the argument in [CDM1] was to prove that a natural pairing was well defined. We have been unable to achieve this proof by any of the arguments that are effective for the case $\mathbb{F}=\{1\}$; it is not hard to see that the problem is equivalent to showing that $\mathrm{Ea}(\mathbb{F})$ is semisimple.

Now we have to modify the argument to deal with the case of any finite set $\mathbb{F}$ with $1 \in \mathbb{F}$. Suppose that $\mathbb{F}$ has $N$ elements, $1, n_{2}, \ldots, n_{N}$. Let $\Phi_{\mathbb{F}}$ be 
the set of all entire functions in $N$ complex variables such that

$$
\left|\varphi\left(z_{1}, \ldots, z_{N}\right)\right| \leq \varpi\left(z_{1}\right) \ldots \varpi\left(z_{N}\right)
$$

and also $\varphi$ satisfies the system of complex partial differential equations

$$
\frac{\partial \varphi}{\partial z_{j}}=\frac{\partial^{n_{j}} \varphi}{\partial z_{1}^{n_{j}}} \quad\left(n_{j} \in \mathbb{F}\right) .
$$

An elaboration of the above argument gives the following theorem.

Theorem 3.3. Let $\mathbb{F}$ be a finite subset of $\mathbb{N}$ with $1 \in \mathbb{F}$. Then the extremal algebra $\mathrm{Ea}(\mathbb{F})$ has dual space $\Phi_{\mathbb{F}}$ and $\mathrm{Ea}(\mathbb{F})$ is isometrically isomorphic to the closed subalgebra of $B\left(\Phi_{\mathbb{F}}\right)$ generated by the hermitian operator $\partial / \partial z_{1}$.

4. Some questions. 1. Does the converse of the Müntz Theorem hold? If $\mathbb{S}$ is a subset of the odds such that $\sum\{1 / n: n \in \mathbb{S}\}<\infty$, do we have $\widehat{\mathbb{S}} \neq 2 \mathbb{N}-1$ ? Similarly for the evens (with a hermitian generator).

2. In all the examples we know, $\widehat{\mathbb{S}}$ is either $\mathbb{S}$ itself, or the union of $\mathbb{S}$ with the evens or the odds (or both). Are these the only possibilities?

3. Is $\mathrm{Ea}(\mathbb{S}) C^{*}$-equivalent if and only if $\widehat{\mathbb{S}} \supseteq 2 \mathbb{N}-1$ ?

4. Is $\mathrm{Ea}(\mathbb{S})$ semisimple for every $\mathbb{S}$ ?

5. Let $\mathbb{F}_{1}, \mathbb{F}_{2}$ be finite subsets of $\mathbb{N}$. Do we have $\mathbb{F}_{1}=\mathbb{F}_{2}$ given that $\operatorname{Ea}\left(\mathbb{F}_{1}\right)$ is isometrically isomorphic to $\mathrm{Ea}\left(\mathbb{F}_{2}\right)$ ? What if we have only a bicontinuous isomorphism?

6. Characterize $\mathbb{S}$ such that $\mathrm{Ea}(\mathbb{S})$ is an amenable Banach algebra.

\section{References}

[BD] F. F. Bonsall and J. Duncan, Numerical Ranges of Operators on Normed Spaces and of Elements of Normed Algebras, Cambridge Univ. Press, London, 1971.

[B1] A. Browder, States, numerical ranges, etc., Proc. Brown Informal Analysis Seminar, 1969.

[B2] - On Bernstein's inequality and the norm of Hermitian operators, Amer. Math. Monthly 78 (1971), 871-873.

[CDM1] M. J. Crabb, J. Duncan and C. M. McGregor, Some extremal problems in the theory of numerical ranges, Acta Math. 128 (1972), 123-141.

[CDM2] - - - - , Some extremal algebras for hermitians, Glasgow Math. J. 43 (2001), $29-38$.

[CDM3] -, 一, 一, The extremal algebra on two hermitians with square 1, ibid., to appear.

[CM1] M. J. Crabb and C. M. McGregor, Numerical ranges of powers of hermitian elements, ibid. 28 (1986), 37-45.

[CM2] -, - Polynomials in a hermitian element, ibid. 30 (1988), 171-176. 
[K] H. König, A functional calculus for Hermitian elements of complex Banach algebras, Arch. Math. (Basel) 28 (1977), 422-430.

[R] W. Rudin, Real and Complex Analysis, third ed., McGraw-Hill, New York, 1987.

Department of Mathematics

University of Glasgow

Glasgow G12 8QW, U.K.

E-mail: M.Crabb@maths.gla.ac.uk

C.McGregor@maths.gla.ac.uk

Department of Mathematics and Statistics

Laval University

Québec, Canada G1K 7P4

E-mail: ransford@mat.ulaval.ca
Department of Mathematical Sciences University of Arkansas

Fayetteville, AR 72701-1201, U.S.A.

E-mail: jduncan@uark.edu 